frequent irritating typographical errors. More importantly, there are several serious omissions which must limit its value. For example, prostacyclin, SRS-A, opiate receptors, encephalins and endorphins are not mentioned, even though they are basic to an understanding of the action of many commonly used drugs. There are other books, competitive in price, which are more comprehensive and up-to-date, and which will probably be preferred.

\section{Principles of Pathology in Surgery}

By K. A. Myers, R. D. Marshall and J. Friedin. Pp. $x i+453$, illustrated. Blackwell Scientific Publications, Oxford, 1980. $£ 12.00$.

This is an interesting student text book written by 3 surgeons at Monash University, Melbourne. Its aim is to present the problems of surgery in terms of pathological principles and in this it succeeds admirably. The introductory chapter, on Focal Lesions, sets out the basic definitions of the broad groups of surgical diseases - tumours, deformities, hernias, etc. - and then demonstrates the effects that may be produced by obstruction of any hollow tube. Further chapters deal with injury, healing, surgical immunology, infections, tumours, haemorrhage and thrombosis, atherosclerosis, body fluid disturbances and shock. Clinical examples are used extensively to illustrate the principles, although no attempt is made to describe specific diseases at length. The place of surgery in dealing with pathological disturbances, such as the excision of tumours, the removal of an obstructing calculus in the ureter, salivary duct or gall-bladder, and the management of arterial occlusive disease is well illustrated.

A feature of the book is the large number of clear line diagrams illustrating pathological conditions (including histology) and their operative management. These, together with a well written and nicely printed text makes this an easy book to enjoy reading.

As Lord Smith points out in his foreword, 'Basic science ... is basic to the understanding of medicine and its study must continue as long as one continues to practise medicine'. A student who reads through this textbook at the beginning of his surgical dressership will stand a good chance of acquiring this useful lifetime habit, and to him it can be recommended with confidence.

\section{Rehabilitation Medicine. The Management of Physical Disabilities}

Edited by P. J. R. Nichols. 2nd edn. Pp. 353. Butterworths, London and Boston, 1980. £19.50.

It is now just over a year since the untimely death of Dr Nichols, and thus an appropriate time to take a dispassionate look at his last major publication. Although this is a multiauthor text book covering the whole range of rehabilitation medicine, it makes the experiment of taking the rehabilitation of rheumatoid arthritis patients as an example for the whole of rehabilitation, and whilst this has good historical antecedents in the development of the combined speciality of rheumatology and rehabilitation, it does have certain practical pitfalls. For example, whilst the advantages of the contraceptive pill are undeniable for a wide range of disabled female patients, its use in patients receiving corticosteroids for rheumatoid arthritis is fraught with unreliability, because of metabolic interactions. There are a number of other ways in which the rehabilitation of rheumatic disease patients differs from that of others, and this weakens the case for this particular experiment in editing.

The book contains valuable introductory chapters on the organization of the rehabilitation services and on physiotherapy and on occupational therapy as such. Not everyone would now agree that ultrasonic therapy is effective solely on account of its heating effect on the tissues, and some of its more specific indications are omitted. However, there is a wise and balanced account of the role of traction and manipulation, stressing the need for controlled trials. Sound advice on the use of scarce physiotherapy resources is given and one is particularly pleased to see the advice that breathing exercises should be restricted to patients at special risk and not used as a routine pre-operative procedure. The chapter on the rehabilitation of patients with rheumatoid arthritis recommends central heating by off-peak electric storage heaters, as being the most economic. This was certainly the case at the time this view was expressed and it is also of interest that the value of showers as compared with ordinary baths is not mentioned - a very British view that would not find favour in Europe or the United States. A greater stress on the value of the Resettlement Clinic, based on a department of rheumatology and rehabilitation, also serving other units, particularly neurology and orthopaedics, should perhaps have been included. The use of brightly coloured warning cards attached to the case notes of patients with atlanto-axial subluxation might be considered for inclusion in a subsequent edition, and the long-term functional results of the MacIntosh partial prosthesis for the severely damaged rheumatoid knee are such as to suggest that the author's enthusiasm for this procedure may now be considered questionable. It certainly renders the operative field less favourable for a subsequent total knee-replacement operation. There is also surprisingly little in this chapter on the role of surgery in the rehabilitation of the rheumatoid foot.

There follows chapters on pain in the neck and shoulders, backache and osteo-arthrosis, which contain a valuable summary of current opinion. One is, however, somewhat depressed to read on page 80 that 'clinical features, methods of examination and measurement and modes of treatment are not amenable to standardisation'. This comment in relation to shoulder pain in particular is an unfortunate and unnecessary counsel of despair and were it to be taken seriously might prejudice the very necessary attempts to research in depth the vast range of acute conditions from superspinatus tendonitis to lumbar root pain, which cause such massive absenteeism from work and consume so great an amount of specialist time. In fact, as was shown for instance by Richardson in his Ernest Fletcher memorial lecture several years ago, if the physical signs are elicited in a standard fashion and vague diagnostic categories avoided, scientific evaluation of treatment becomes possible, and in fact procedures for proper trials of physical forms of treatment are now being evolved in a number of centres.

The chapter on disorders of peripheral nerves by Wynn Parry is of a very high quality and differs from other chapters in being illustrated by a number of most informative case histories. This is perhaps the point at which to remark on one other extraordinary feature of this book, and that is the total absence of illustrations or figures of any kind, even the simplest of line drawings being omitted. On the whole the authors have risen to the occasion exceedingly well and in this chapter particularly so.

The chapter on the rehabilitation of stroke is comprehensive, but would be improved by inclusion of more recent data on diagnostic criteria and techniques and the next edition might, with profit, include an account of the newer work on cerebral dominance in relation to particular functions and the implications that this may have for stroke rehabilitation. The section on wheel-chairs suffers badly from the lack of illustrations, particularly for anyone coming fresh to the field of rehabilitation, who will find it difficult to visualize many of the structures described. This chapter will only make sense when the book is taken along as a guide to learning when attending a wheel-chair clinic.

This book is notable for 3 chapters at the end, which point the way forward in rehabilitation. There is one on psychological and behavioural aspects, one on sexual counselling for the disabled and one on the design of rehabilitation research 